\title{
Localized direct boundary-domain integro-differential formulations for incremental elasto-plasticity of inhomogeneous body
}

\author{
S.E. Mikhailov \\ Division of Mathematics, Glasgow Caledonian University, Glasgow, G4 OBA, UK
}

Received 20 June 2005; accepted 7 July 2005

\begin{abstract}
A quasi-static mixed boundary value problem of incremental elasto-plasticity for a continuously inhomogeneous body is considered. Using the two-operator Green-Betti formula and the fundamental solution of a reference homogeneous linear elasticity problem, with frozen initial or tangent elastic coefficients, a boundary-domain integro-differential formulation of the elasto-plastic problem is presented, with respect to the displacement rates and their gradients. Using a cut-off function approach, the corresponding localized parametrix of the reference problem is constructed to reduce the elasto-plastic problem to a nonlinear localized boundary-domain integro-differential equation. Algorithms of meshbased and mesh-less discretizations are presented resulting in sparsely populated systems of nonlinear algebraic equations for the displacement increments.
\end{abstract}

(C) 2005 Elsevier Ltd. All rights reserved.

Keywords: Incremental elasto-plasticity; Functionally graded materials; Variable coefficients; United formulation; Partly segregated formulation; Integrodifferential equation; Localization; Mesh-based discretization; Mesh-less discretization

\section{Introduction}

It is well known, see e.g. [1-5], that using a fundamental solution of the reference linear elastic problem, with the initial elastic coefficients, in the corresponding Green-Betty identity, an elasto-plastic problem for a homogeneous body can be reduced to a non-linear boundary-domain integral equation for increments, with singular domain integrals and hyper-singular boundary integrals. Using an iteration procedure, one can further reduce the problem to a sequence of purely boundary integral equations.

However, the reference fundamental solution is usually highly non-local, which leads after discretization to a system of algebraic equations with a dense matrix. Moreover, the fundamental solution is generally not available in an explicit form if the material is inhomogeneous (functionally graded), i.e. the coefficients of the reference problem vary in space. In addition, an evaluation of the singular volume integrals and hyper-singular domain integrals increases the complexity of the numerical code and reduces its accuracy.

To prevent similar difficulties for linear scalar (heat transfer) equation in inhomogeneous medium, some

E-mail address: s.mikhailov@gcal.ac.uk. parametrices (Levi functions) localized by cut-off function multiplication were constructed and implemented in [6]. This reduced the linear Boundary Value Problem (BVP) with variable coefficient to a linear Localized Boundary-Domain Integral or Integro-Differential equation (LBDIE or LBDIDE), which leaded after a mesh-based or mesh-less discretization to a linear algebraic system with a sparse matrix. Some meshbased numerical implementations of the linear LBDIE were presented in $[7,8]$. Somewhat different LBDI(D)E formulations and numerical realizations were presented in $[9,10]$.

Extending approach of [6], the mixed BVP for a second order scalar nonlinear (quasi-linear) elliptic PDE with the variable coefficient dependent on the unknown solution was reduced in [11,12], to quasi-linear LBDIDEs. For the case when the variable coefficient depends also on the BVP solution gradient, some quasi-linear two-operator LBDIDEs were obtained in [13,12]. The approach was extended in $[14,15]$ to the mixed BVP for the system of quasi-linear partial differential equations of physically nonlinear elasticity (with small deformation gradients) for continuously inhomogeneous body. Another approach based on local parametrices that are Green functions for an auxiliary problem on local spherical domains, was used in [16-19] reducing some linear and nonlinear problems for a body with a special inhomogeneity to local boundary-domain integral equations solved numerically by the mesh-less methods. 
In this paper, extending results of [20], we apply the localization approach of $[6,11-15]$ to the mixed BVP for the system of incremental elasto-plasticity (with small deformation gradients) for continuously inhomogeneous body. First, we present reduction of the BVP to either a united direct twooperator nonlinear BDIDE of the second kind for the displacement rates (or increments) or to a partly segregated direct two-operator nonlinear BDIDE of the third kind for the displacement rates (or increments) and unknown boundary tractions. The equations include at most the first derivatives of the unknown solution, weakly singular integrals over the domain and at most Cauchy-type singular integrals over the boundary. Then we present a localized version of the BDIDEs and describe their mesh-based and mesh-less discretizations.

\section{Elements of incremental elasto-plasticity}

Let $u(x)=u_{\mathrm{i}}(x)$ be the displacement vector in $\mathbb{R}^{n}$, where $n$ is either 2 or 3 ;

$\varepsilon_{i j}(x)=\left[u_{i, j}(x)+u_{j, i}(x)\right] / 2$

be the strain tensor, $\sigma_{i j}(x)$ be the stress tensor. The comma in front of a superscript means derivative with respect to the corresponding coordinate. Summation in repeated indices is further supposed from 1 to $n$ unless stated otherwise.

Constitutive equations of the incremental elasto-plasticity can be written as

$$
\begin{aligned}
\varepsilon_{i j}(x)= & \varepsilon_{i j}^{e}(x)+\varepsilon_{i j}^{p}(x), \\
\sigma_{i j}(x)= & a_{i j k l}^{e}(x) \varepsilon_{k l}^{e}(x), \\
\dot{\varepsilon}_{i j}^{p}(x)= & G_{i j}(\varepsilon(x), \sigma(x), x) G_{k l}(\varepsilon(x), \sigma(x), x) \dot{\sigma}_{k l}(x) \\
& \times H\left[G_{p q}(\varepsilon(x), \sigma(x), x) \dot{\sigma}_{p q}(x)\right]
\end{aligned}
$$

Here the over dot means derivative with respect to time; $a_{i j k l}^{e}(x)$ is a known function of the coordinates $x$, such that $a_{i j k l}^{e}(x)=a_{j i k l}^{e}(x)=a_{i j k}^{e}(x)=a_{k l i j}^{e}(x)$ and

$\varepsilon_{k l} a_{i j k l}^{e}(x) \varepsilon_{k l} \geq 0 \quad \forall \varepsilon_{k l}$;

the symmetric tensor $G_{i j}(\varepsilon, \sigma, x)$ is a known functional on the loading history, $\{\varepsilon\}$ and $\{\sigma\}$, and of the current state, $\varepsilon$ and $\sigma$, at point $x$; the multiplier with the Heaviside function,

$H[z]:=\left\{\begin{array}{ll}1, & z \geq 0 \\ 0, & z<0\end{array}\right.$,

is employed in (4) to ensure that the plastic strain increment is zero during unloading, which follows particularly from the Drucker principle in plasticity.

Relations (1)-(4) and condition (5) allow to express the stress rate in terms of the displacement rate gradients,

$\dot{\sigma}_{i j}(x)=a_{i j k l}(\sigma(x), \varepsilon(x), \nabla \dot{u}(x), x) \dot{u}_{k, l}(x)$,

where $a_{i j k l}(\sigma, \varepsilon, \nabla \dot{u}, x)$ is the current tangent moduli tensor, given by the formula

$$
\begin{aligned}
& a_{i j k l}(\sigma, \varepsilon, \nabla \dot{u}, x): \\
& \quad=a_{i j k l}^{e}(x)-g_{i j}(\sigma, \varepsilon, x) g_{k l}(\sigma, \varepsilon, x) H\left[g_{p q}(\sigma, \varepsilon, x) \dot{u}_{p, q}(x)\right], \\
& g_{i j}(\sigma, \varepsilon, x):=\frac{a_{i j k l}^{e}(x) G_{k l}(\sigma, \varepsilon, x)}{\sqrt{1+G_{p q}(\sigma, \varepsilon, x) a_{p q r s}^{e}(x) G_{r s}(\sigma, \varepsilon, x)}}
\end{aligned}
$$

The counterparts of relation (7) without the Heaviside function multiplier, commonly used in the boundary(-domain) integral equations (see, e.g. [2]), are justified, strictly speaking, only for plastic processes without unloading. Note that this multiplier makes relation (6) and consequently all the formulations below, nonlinear with respect to the displacement rate.

Substituting (6) in the time derivative of the equilibrium equation,

$\dot{\sigma}_{i j, j}=\dot{f}_{i}$,

where $f_{i}(x)$ is a known volume force vector (taken with the opposite sign), and in the traction boundary conditions, we arrive at the following mixed boundary-value problem of incremental elasto-plasticity for a bounded inhomogeneous body $\Omega \in \mathbb{R}^{n}$,

$$
\begin{aligned}
{\left[L_{i k}(\sigma, \varepsilon, \dot{u}) \dot{u}_{k}\right](x): } & =\frac{\partial}{\partial x_{j}}\left[a_{i j k l}(\sigma(x), \varepsilon(x), \nabla \dot{u}(x), x) \frac{\partial \dot{u}_{k}(x)}{\partial x_{l}}\right] \\
& =\dot{f}_{i}(x), \quad x \in \Omega, \\
\dot{u}_{i}(x)=\check{u}_{i}(x), \quad x & \in \partial_{\mathrm{D}} \Omega, \\
{\left[T_{i k}(\sigma, \varepsilon, \dot{u}) \dot{u}_{k}\right](x): } & =a_{i j k l}(\sigma(x), \varepsilon(x), \nabla \dot{u}(x), x) \frac{\partial \dot{u}_{k}(x)}{\partial x_{l}} n_{j}(x) \\
& =\check{t}_{i}(x), \quad x \in \partial_{\mathrm{N}} \Omega .
\end{aligned}
$$

Here $n_{i}(x)$ is an outward normal vector to the boundary $\partial \Omega$; $[T(\sigma, \varepsilon, \dot{u}) \dot{u}](x)=\left[T_{i k}(\sigma, \varepsilon, \dot{u}) \dot{u}_{k}\right](x)$ is the traction rate vector at a boundary point $x$, while $T(\sigma, \varepsilon, \dot{u})=T_{i k}(\sigma, \varepsilon, \dot{u})$ is the nonlinear traction differential operator; $\check{u}(x)$ and $\breve{t}(x)$ are known displacement rate and traction rate vectors on the parts $\partial_{\mathrm{D}} \Omega$ and $\partial_{\mathrm{N}} \Omega$ of the boundary, respectively. The left hand sides of elasto-plasticity BVP (10)-(12) do not include time explicitly and one may replace there the rates $\dot{u}_{k}$ by the differentials $\Delta u_{k}=\dot{u}_{k} \Delta t$.

If $n=2, \mathrm{BVP}$ (10)-(12) describes the 2D elasto-plasticity problems in the plane strain state. In the plane stress state, the tensor $a_{i j k l}$ is to be replaced by a corresponding combination of its components.

For brevity, we will drop the arguments $\sigma$ and $\varepsilon$ of the functionals $g_{k l}(\sigma(x), \varepsilon(x), x), a_{i j k l}(\sigma(x), \varepsilon(x), \nabla \dot{u}(x), x)$ and operators $L(\sigma, \varepsilon, \dot{u}), T(\sigma, \varepsilon, \dot{u})$ in the equations below but their dependence on the process history and the actual stress and strain tensors will be meant nevertheless. 


\section{Two-Operator Green-Betti identities and direct BDIDEs of incremental elasto-plasticity}

\subsection{Green-Betti identities}

Let us fix a point $y$ and consider the following reference differential operators of linear elasticity with some constant (frozen at the point $y$ ) coefficients, $a_{i j k l}^{*}(y)$,

$\left[L_{i k}^{(y) *} v_{k}\right](x):=\frac{\partial}{\partial x_{j}}\left[a_{i j k l}^{*}(y) \frac{\partial v_{k}(x)}{\partial x_{l}}\right]$

$\left[T_{i k}^{(y) *} v_{k}\right](x):=a_{i j k l}^{*}(y) \frac{\partial v_{k}(x)}{\partial x_{l}} n_{j}(x)$.

Under the reference elastic coefficients $a_{i j k l}^{*}(y)=a_{i j k l}^{*}(\nabla \dot{u}(y), y)$, one can understand either the initial elastic moduli $a_{i j k l}^{e}(y)$ independent of the strain-stress history and the current strain rate, or the current tangent moduli $a_{i j k l}(\nabla \dot{u}(y), y)$ dependent on both the strain-stress history and the current strain rate. The same character of dependence on (or independence of) the strain-stress history and the current strain rate will then remain for all asterisk variables and operators below. The particular choice of $a_{i j k l}^{*}(\nabla \dot{u}(y), y)$ leads to two different versions of the integro-differential equations.

Integrating by parts, we have the first Green identities for the differential operators $[L(\dot{u}) \dot{u}](x)=\left[L_{i k}(\dot{u}) \dot{u}_{k}\right](x)$ and $\left[L^{(y) *} v\right](x)=\left[L_{i k}^{(y) *} v_{k}\right](x)$,

$$
\begin{aligned}
& \int_{\Omega} v_{i}(x)\left[L_{i k}(\dot{u}) \dot{u}_{k}\right](x) \mathrm{d} \Omega(x) \\
& =\int_{\partial \Omega} v_{i}(x)\left[T_{i k}(\dot{u}) \dot{u}_{k}\right](x) \mathrm{d} \Gamma(x) \\
& \quad-\int_{\Omega} \frac{\partial v_{i}(x)}{\partial x_{j}} a_{i j k l}(\nabla \dot{u}(x), x) \frac{\partial \dot{u}_{k}(x)}{\partial x_{l}} \mathrm{~d} \Omega(x), \\
& \int_{\Omega} \dot{u}_{i}(x)\left[L_{i k}^{(y) *} v_{k}\right](x) \mathrm{d} \Omega(x) \\
& =\int_{\partial \Omega} \dot{u}_{i}(x)\left[T_{i k}^{(y) *} v_{k}\right](x) \mathrm{d} \Gamma(x) \\
& \quad-\int_{\Omega} \frac{\partial \dot{u}_{i}(x)}{\partial x_{j}} a_{i j k l}^{*}(\nabla \dot{u}(y), y) \frac{\partial v_{k}(x)}{\partial x_{l}} \mathrm{~d} \Omega(x),
\end{aligned}
$$

where $\dot{u}(x)$ and $v(x)$ are arbitrary vector-functions for that the operators and integrals in the above expressions have sense. Subtracting the identities from each other and taking into account the symmetry of the tensor $a_{i j k l}$, we derive the twooperator second Green-Betti identity,

$$
\begin{gathered}
\int_{\Omega}\left\{\dot{u}(x)\left[L^{(y) *} v\right](x)-v(x)[L(\dot{u}) \dot{u}](x)\right\} \mathrm{d} \Omega(x) \\
=\int_{\partial \Omega}\left\{\dot{u}(x)\left[T^{(y) *} v\right](x)-v(x)[T(\dot{u}) \dot{u}](x)\right\} \mathrm{d} \Gamma(x) \\
+\int_{\Omega}[\nabla v(x)] \tilde{a}(\nabla \dot{u} ; x, y) \nabla \dot{u}(x) \mathrm{d} \Omega(x),
\end{gathered}
$$

where

$$
\begin{aligned}
\tilde{a}(\nabla \dot{u} ; x, y)= & \tilde{a}_{i j k l}(\nabla \dot{u}(x), \nabla \dot{u}(y), x, y): \\
= & a_{i j k l}(\nabla \dot{u}(x), x)-a_{i j k l}^{*}(\nabla \dot{u}(y), y) \\
= & a_{i j k l}^{e}(x)-a_{i j k l}^{*}(\nabla \dot{u}(y), y) \\
& -g_{i j}(x) g_{k l}(x) H\left[g_{p q}(x) \dot{u}_{p, g}(x)\right] .
\end{aligned}
$$

Note that for a homogeneous material $a_{i j k l}^{e}(x)=a_{i j k l}^{e}(y)$, which implies $\tilde{a}(\nabla \dot{u} ; x, y)$ is non-zero only when $x$ or $y$ if $\left.a_{i j k l}^{*}(y)=a_{i j k l}(y)\right]$ is in the plasticity zone with active loading. If moreover $L(\dot{u})=L^{(y) *}$, i.e. $L(\dot{u})$ is a linear homogeneous elasticity operator, then the last domain integral disappears in (13), which thus degenerates into the classical second GreenBetti identity.

For a fixed $y$, let $F^{(y) *}(x, y)=F_{k m}^{(y) *}(x, y)$ be a fundamental solution for the linear differential operator $\left[L_{i k}^{(y) *} v_{k}\right](x)$ with constant coefficients, i.e.

$\left[L_{i k}^{(y) *} F_{k m}^{(y) *}(\cdot, y)\right](x):=a_{i j k l}^{*}(y) \frac{\partial^{2} F_{k m}^{(y) *}(x, y)}{\partial x_{j} \partial x_{l}}=\delta_{i m} \delta(x-y)$,

where $\delta_{i m}$ is the Kronecker symbol and $\delta(x-y)$ is the Dirac delta-function.

If the material is isotropic and $a_{i j k l}^{*}(x)=a_{i j k l}^{e}(y)$, then

$a_{i j k l}^{*}(y)=\lambda^{*}(y) \delta_{i j} \delta_{k l}+\mu^{*}(y)\left(\delta_{i k} \delta_{j l}+\delta_{i l} \delta_{j k}\right)$.

In this case, $F_{i m}^{(y) *}(x, y)$ is the Kelvin-Somigliana solution,

$F_{i m}^{(y) *}(x, y)=\frac{-1}{4 \pi}\left\{\frac{-\delta_{i m} \ln r-r_{, i} r_{, m}}{\lambda^{*}(y)+2 \mu^{*}(y)}+\frac{-\delta_{i m} \ln r+r_{, i} r_{, m}}{\mu^{*}(y)}\right\}$

for the plane strain state; for the plane stress, $\lambda^{*}$ in (14) and (15) should be replaced by $2 \lambda^{*} \mu^{*} /\left(\lambda^{*}+2 \mu^{*}\right)$. In the $3 \mathrm{D}$ case,

$F_{i m}^{(y) *}(x, y)=\frac{-1}{8 \pi r}\left\{\frac{\delta_{i m}-r_{, i} r_{, m}}{\lambda^{*}(y)+2 \mu^{*}(y)}+\frac{\delta_{i m}+r_{, i} r_{, m}}{\mu^{*}(y)}\right\}$

Here $r:=\sqrt{\left(x_{i}-y_{i}\right)\left(x_{i}-y_{i}\right)}, r_{, i}:=\partial r / \partial x_{i}=\left(x_{i}-y_{i}\right) / r$. For $a_{i j k l}^{*}$ $(x)=a_{i j k l}(y)$ or for an initially anisotropic material, the fundamental solution can be written down in an analytical form for arbitrary anisotropy in the 2D case and for some particular anisotropy in the 3D case; otherwise, it can be expressed, e.g. as a linear integral over a circle [21-23].

Assuming $\dot{u}(x)$ is a solution of nonlinear system (10) and using the fundamental solution $F^{(y)^{*}}(x, y)$ as $v(x)$ in the Green identity (13), we obtain, similar to the linear homogeneous elasticity (see, e.g. [1-5]) or partial differential equations with variable coefficients [24], the following non-linear twooperator third Green identity, 


$$
\begin{aligned}
& c(y) \dot{u}(y)-\int_{\partial \Omega} \dot{u}(x)\left[T^{(y) *} F^{(y) *}(\cdot, y)\right](x) \mathrm{d} \Gamma(x) \\
& +\int_{\partial \Omega} F^{(y) *}(x, y)[T(\dot{u}) \dot{u}](x) \mathrm{d} \Gamma(x) \\
& -\int_{\Omega}\left[\nabla^{(x)} F^{(y) *}(x, y)\right] \tilde{a}(\nabla \dot{u} ; x, y) \nabla \dot{u}(x) \mathrm{d} \Omega(x) \\
& =\int_{\Omega} F^{(y) *}(x, y) f(x) \mathrm{d} \Omega(x),
\end{aligned}
$$

where the coefficient tensor $c(y)=c_{i m}(y)$ is such that $c_{i m}(y)=$ $\delta_{i m}$ if $y \in \Omega ; c_{i m}(y)=0$ if $y \notin \bar{\Omega} ; c_{i m}(y)=(1 / 2) \delta_{i m}$ if $y$ is a smooth point of the boundary $\left(\Omega\right.$; and $c_{i m}(y)=c_{i m}\left(a^{*}(y), \alpha(y)\right)$ is a function of the reference tensor $a^{*}(y)$ and the interior space angle $\alpha(y)$ at a corner point $y$ of the boundary $\partial \Omega$.

\subsection{United nonlinear two-operator BDIDE}

Substituting boundary conditions (11) and (12) into the integrands of identity (17) and using it at $y \in \bar{\Omega}$, we arrive at a united nonlinear two-operator Boundary-Domain IntegroDifferential Equation, BDIDE, of the second kind for $\dot{u}(x)$ at $x \in \bar{\Omega}$

$$
\begin{aligned}
& c(y) \dot{u}(y)-\int_{\partial_{\mathrm{N}} \Omega} \dot{u}(x)\left[T^{(y) *} F^{(y)^{*}}(\cdot, y)\right](x) \mathrm{d} \Gamma(x) \\
& +\int_{\partial_{\mathrm{D}} \Omega} F^{(y)^{*}}(x, y)[T(\dot{u}) \dot{u}](x) \mathrm{d} \Gamma(x) \\
& -\int_{\Omega}\left[\nabla^{(x)} F^{(y) *}(x, y)\right] \tilde{a}(\nabla \dot{u} ; x, y) \nabla \dot{u}(x) \mathrm{d} \Omega(x)=\mathscr{F}(y), y \in \bar{\Omega},
\end{aligned}
$$

$$
\begin{aligned}
\mathscr{F}(y):= & \int_{\partial_{\mathrm{D}} \Omega} \check{u}(x)\left[T^{(y) *} F^{(y) *}(\cdot, y)\right](x) \mathrm{d} \Gamma(x) \\
& -\int_{\partial_{\mathrm{N}} \Omega} F^{(y) *}(x, y) \check{t}(x) \mathrm{d} \Gamma(x) \\
& +\int_{\Omega} F^{(y) *}(x, y) f(x) \mathrm{d} \Omega(x) .
\end{aligned}
$$

\subsection{Partly segregated nonlinear two-operator BDIDE}

On the other hand, introducing a new vector variable $\tilde{t}(x)$ instead of the unknown traction vector rate $[T(\dot{u}) \dot{u}](x)$ at $x \in \partial_{\mathrm{D}} \Omega$ and substituting $\breve{u}(y)$ also for the out-of-integral term at $y \in \partial_{\mathrm{D}} \Omega$, we may reduce the incremental plasticity problems (10)-(12) to the following partly segregated BDIDE of the third kind for $\dot{u}(x)$ at $x \in \Omega \cup \partial_{\mathrm{N}} \Omega$ and $\tilde{t}(x)$ at $x \in \partial_{\mathrm{D}} \Omega$,

$$
\begin{aligned}
& c^{0}(y) \dot{u}(y)-\int_{\partial_{N} \Omega} \dot{u}(x)\left[T^{(y) *} F^{(y) *}(\cdot, y)\right](x) \mathrm{d} \Gamma(x) \\
& +\int_{\partial_{D} \Omega} F^{(y) *}(x, y)[T(\dot{u}) \dot{u}](x) \mathrm{d} \Gamma(x) \\
& -\int_{\Omega}\left[\nabla^{(x)} F^{(y) *}(x, y)\right] \tilde{a}(\nabla \dot{u} ; x, y) \nabla \dot{u}(x) \mathrm{d} \Omega(x)=\mathcal{F}^{0}(y), \quad y \in \bar{\Omega},
\end{aligned}
$$

$\mathcal{F}^{0}(y):=\left[c^{0}(y)-c(y)\right] \breve{u}(y)+\mathscr{F}(y), \quad y \in \Omega \cup \partial \Omega$,

$c^{0}(y)=\left\{\begin{array}{ll}0, & \text { if } y \in \partial_{D} \Omega ; \\ c(y), & \text { if } y \in \Omega \cup \partial_{\mathrm{N}} \Omega\end{array}\right.$.

BDIDEs (18) and (19) include at most the first space derivatives of the unknown solution $\dot{u}(x)$, both directly in the domain integral term in the left hand side and through the coefficient $a(\nabla \dot{u}(x), x, y)$ in the operator $T(\dot{u})$ and in the function $\tilde{a}(\nabla \dot{u} ; x, y)$. The function $\left[\nabla^{(x)} F^{(y)^{*}}(x, y)\right]$ is at most weakly singular in $\Omega$, and assuming that $\tilde{a}(\nabla \dot{u} ; x, y)$ is bounded as $x \rightarrow y$, we obtain that the domain integral is a smoothing operator for $\nabla \dot{u}(y)$. The boundary integrals have at most the Cauchy-type singularity.

The right hand side of BDIDEs (18) and (19) are independent of $\nabla \dot{u}$ if the reference tensor $a^{*}$ is chosen as the initial elastic tensor $a^{e}$. Otherwise, when $a^{*}$ is chosen as the tangent stiffness tensor $a$, the right hand side dependence on $\nabla \dot{u}$ will be present.

Some other (e.g. segregated) BDIDEs can be obtained if one also considers the unknown boundary displacement rate $\dot{u}$ on $\partial_{N} \Omega$ as a new vector variable formally segregated from $\dot{u}$ in $\Omega$, or applies the boundary traction operator to (18) or (19).

Each of BDIDEs (18) and (19) can be reduced after some discretization to a system of nonlinear algebraic equation and solved numerically. The system will include unknowns not only on the boundary but also at internal points. Moreover, since the fundamental solutions, c.f. (15) and (16), are highly non-local, the matrix of the system will be fully populated and this makes its numerical solution more expensive. To avoid this difficulty, we present below some ideas of constructing localized parametrices and consequently Localized BDIDEs (LBDIDEs).

\section{Localized parametrix and LBDIDEs of incremental elasto-plasticity}

\subsection{Localization}

Let $\chi(x, y)$ be a cut-off function, such that $\chi(y, y)=1$ and $\chi(x, y)=0$ at $x$ not belonging to closure of an open localization domain $\omega(\mathrm{y})$ (a vicinity of $y$ ), see Fig. 1, and let $P_{\omega}^{(y) *}(x, y)=\chi(x, y) F^{(y) *}(x, y)$. 


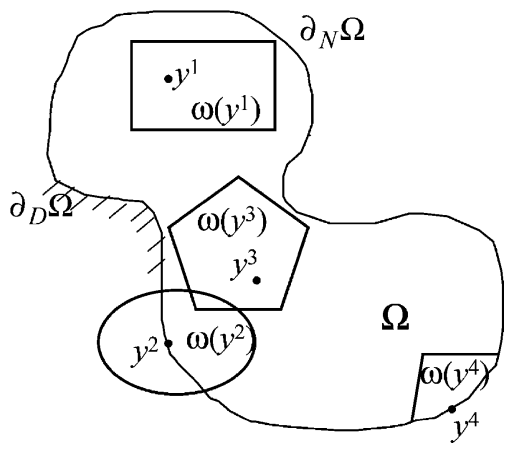

Fig. 1. Body $\Omega$ with localization domains $\omega\left(y^{i}\right)$.

The simplest example is

$\chi(x, y)=\left\{\begin{array}{ll}1, & x \in \bar{\omega}(22) \\ 0, & x \notin \bar{\omega}\end{array} \Rightarrow\right.$
$P_{\omega}^{(y) *}(x, y)= \begin{cases}F^{(y) *}(x, y), & x \in \bar{\omega}(y) \\ 0, & x \notin \bar{\omega}(y)\end{cases}$

Other examples of the cut-off functions having different smoothness are presented in [6,12] for some shapes of the localization domains $\omega$.

Then $P_{\omega}^{(y) *}(x, y)$ is a localized parametrix (localized Levi's function) of the linear operator $L^{(y)^{*}}$, i.e.

$L_{i k}^{(y) *} P_{k m \omega}^{(y) *}(x, y)=\delta_{i m} \delta(x-y)+R_{i m \omega}^{(y) *}(x, y)$,

where the remainder

$$
\begin{aligned}
R_{i m \omega}^{(y) *} & =-L_{i k}^{(y) *}\left((1-\chi) F_{k m}^{(y) *}\right) \\
& =a_{i j k l}^{*}(y)\left[F_{k m}^{(y) *} \frac{\partial^{2} \chi}{\partial x_{j} \partial x_{l}}+\frac{\partial F_{k m}^{(y) *}}{\partial x_{j}} \frac{\partial \chi}{\partial x_{l}}+\frac{\partial F_{k m}^{(y) *}}{\partial x_{l}} \frac{\partial \chi}{\partial x_{j}}\right]
\end{aligned}
$$

is at most weakly singular at $x=y$ if $\chi$ is smooth enough on $\bar{\omega}(y)$. The parametrix $P_{\omega}^{(y) *}(x, y)$ has the same singularity as $F^{(y) *}(x, y)$ at $x=y$. Both $P_{\omega}^{(y) *}(x, y)$ and $R_{\omega}^{(y) *}(x, y)$ are localized (non-zero) with respect to $x$ only on $\omega(y)$.

Suppose $\chi(x, y)$ is smooth in $x \in \bar{\omega}(y)$ but not necessarily zero at $x \in \partial \omega(y)$, c.f. (22). Then $P_{\omega}^{(y) *}(x, y)$ is a discontinuous localized parametrix at $x \in \mathbb{R}^{n}$, and $P_{\omega}^{(y) *}(x, y)=R_{\omega}^{(y) *}(x, y)=0$ if $x \notin \bar{\omega}(y)$. Substituting $P_{\omega}^{(y) *}(x, y)$ for $v(x)$ in (13), replacing $\Omega$ by the intersection $\omega(y) \cap \Omega$ and repeating the same arguments as for the fundamental solution above, we arrive at the localized parametrix-based two-operator third Green identity on $\bar{\omega}(y) \cap \bar{\Omega}$,

$$
\begin{aligned}
& c(y) \dot{u}(y)-\int_{\bar{\omega}(y) \cap \partial \Omega}\left\{\dot{u}(x)\left[T^{(y) *} P_{\omega}^{(y) *}(\cdot, y)\right](x)-P_{\omega}^{(y) *}(x, y)\right. \\
& \times[T(\dot{u}) \dot{u}](x)\} \mathrm{d} \Gamma(x)-\int_{\Omega \cap \partial \omega(y)}\left\{\dot{u}(x)\left[T^{(y) *} P_{\omega}^{(y) *}(\cdot, y)\right](x)\right. \\
& \left.-P_{\omega}^{(y) *}(x, y)[T(\dot{u}) \dot{u}](x)\right\} \mathrm{d} \Gamma(x) \\
& -\int_{\omega(y) \cap \Omega}\left\{\left[\nabla^{(x)} P_{\omega}^{(y) *}(x, y)\right] \tilde{a}(\nabla \dot{u} ; x, y) \nabla \dot{u}(x)\right. \\
& \left.-R_{\omega}^{(y) *}(x, y) \dot{u}(x)\right\} \mathrm{d} \Omega(x) \\
& =\int_{\omega(y) \cap \Omega} P_{\omega}^{(y) *}(x, y) f(x) \mathrm{d} \Omega(x) .
\end{aligned}
$$

The second term in the last integral in the left hand side of (23) disappears if $\chi(x, \mathrm{y})$ is given by (22).

\subsection{United nonlinear two-operator LBDIDE}

Substituting boundary conditions (11) and (12) into the integral terms of (23) and employing it at $y \in \bar{\Omega}$, we arrive at the united formulation of nonlinear two-operator Localized Boundary-Domain Integro-Differential Equation (LBDIDE) of the second kind, for $\dot{u}(x), x \in \bar{\Omega}$,

$$
\begin{aligned}
& c(y) \dot{u}(y)-\int_{\bar{\omega}(y) \cap \partial_{\mathrm{N}} \Omega} \dot{u}(x)\left[T^{(y) *} P_{\omega}^{(y) *}(\cdot, y)\right](x) \mathrm{d} \Gamma(x) \\
& +\int_{\bar{\omega}(y) \cap \partial_{D} \Omega} P_{\omega}^{(y) *}(x, y)[T(\dot{u}) \dot{u}](x) \mathrm{d} \Gamma(x) \\
& -\int_{\Omega \cap \partial \omega(y)} \dot{u}(x)\left[T^{(y) *} P_{\omega}^{(y) *}(\cdot, y)\right](x) \mathrm{d} \Gamma(x) \\
& +\int_{\Omega \cap \partial \omega(y)} P_{\omega}^{(y) *}(x, y)[T(\dot{u}) \dot{u}](x) \mathrm{d} \Gamma(x) \\
& -\int_{\omega(y) \cap \Omega}\left[\nabla^{(x)} P_{\omega}^{(y) *}(x, y)\right] \tilde{a}(\nabla \dot{u} ; x, y) \nabla \dot{u}(x) \mathrm{d} \Omega(x)+ \\
& \int_{\omega(y) \cap \Omega}^{R_{\omega}^{(y) *}(x, y) \dot{u}(x) \mathrm{d} \Omega(x)=\mathcal{F}_{\omega}(y), \quad y \in \bar{\Omega},}
\end{aligned}
$$

$$
\begin{aligned}
\mathscr{F}_{\omega}(y):= & \int_{\bar{\omega}(y) \cap \partial_{D} \Omega} \check{u}(x)\left[T^{(y) *} P_{\omega}^{(y) *}(\cdot, y)\right](x) \mathrm{d} \Gamma(x) \\
& -\int_{\bar{\omega}(y) \cap \partial_{N} \Omega} P_{\omega}^{(y) *}(x, y) \check{t}(x) \mathrm{d} \Gamma(x) \\
& +\int_{\omega(y) \cap \Omega} P_{\omega}^{(y) *}(x, y) f(x) \mathrm{d} \Omega(x) .
\end{aligned}
$$




\subsection{Partly segregated nonlinear two-operator LBDIDE}

On the other hand, substituting $\check{u}(y)$ also for the out-ofintegral term $u(y)$ at $y \in \partial \Omega_{\mathrm{D}}$ and introducing a new vector variable $\tilde{t}(x)$ for the unknown traction vector $[T(u) u](x)$ at $x \in \partial \Omega_{\mathrm{D}}$ in (25), one can reduce BVP (10)-(12) to the following partly segregated nonlinear two-operator direct LBDIDE of the third kind, for $u(x)$ at $x \in \Omega \cup \partial_{\mathrm{N}} \Omega$ and $\tilde{t}(x)$ at $x \in \partial \Omega_{\mathrm{D}}$,

$$
\begin{aligned}
& c^{0}(y) \dot{u}(y)-\int_{\bar{\omega}(y) \cap \partial_{N} \Omega} \dot{u}(x)\left[T^{(y) *} P_{\omega}^{(y) *}(\cdot, y)\right](x) \mathrm{d} \Gamma(x) \\
& +\int_{\bar{\omega}(y) \cap \partial_{D} \Omega} P_{\omega}^{(y) *}(x, y) \tilde{t}(x) \mathrm{d} \Gamma(x) \\
& -\int_{\Omega \cap \partial \omega(y)} \dot{u}(x)\left[T^{(y) *} P_{\omega}^{(y) *}(\cdot, y)\right](x) \mathrm{d} \Gamma(x) \\
& +\int_{\Omega \cap \partial \omega(y)} P_{\omega}^{(y) *}(x, y)[T(\dot{u}) \dot{u}](x) \mathrm{d} \Gamma(x) \\
& -\int_{\omega(y) \cap \Omega}\left[\nabla^{(x)} P_{\omega}^{(y) *}(x, y)\right] \tilde{a}(\nabla \dot{u} ; x, y) \nabla \dot{u}(x) \mathrm{d} \Omega(x) \\
& +\int_{\omega(y) \cap \Omega} R_{\omega}^{(y) *}(x, y) \dot{u}(x) \mathrm{d} \Omega(x)=\mathscr{F}_{\omega}^{0}(y), \quad y \in \bar{\Omega}, \\
& \mathscr{F}_{\omega}^{0}(y):=\left[c^{0}(y)-c(y)\right] \check{u}(y)+\mathscr{F}_{\omega}(y) .
\end{aligned}
$$

The very last integrals in the left hand sides of (25) and (27) disappear if $\chi(x, y)$ is given by (22). On the other hand, if a cutoff function $\chi(x, y)$ vanishes at $x \in \partial \omega(y)$ with vanishing normal derivatives, then the integrals along $\Omega \cap \partial \omega(y)$ disappear in (25) and (27).

\section{Discretization of nonlinear two-operator LBDIEs of incremental elasto-plasticity}

The discretization algorithms of LBDIDEs of the incremental elasto-plasticity described below follow mainly the same scheme as in [6-15], although have some special features.

To reduce quasi-linear LBDIDE (25) or (27) to a sparsely populated system of quasi-linear algebraic equations, e.g. by the collocation method, one has to employ a local interpolation or approximation formula for the unknown function $\dot{u}(x)$, for example associated with a mesh-based or mesh-less discretization.

\subsection{Mesh-based discretization}

\subsubsection{Mesh-based interpolation}

Suppose the domain $\Omega$ is covered by a mesh of closures of disjoint domain elements $e_{k}$ with nodes set up at the corners, edges, faces, or inside the elements. Let $J$ be the total number of nodes $x^{i}(i=1,2, \ldots J)$. One can use each node $x^{i}$ as a collocation point for the LBDIDE with a localization domain $\omega\left(x^{i}\right)$. Let the union of closures of the domain elements that
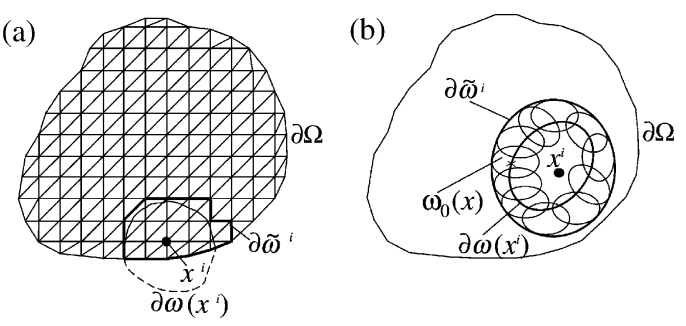

Fig. 2. Localization domain $\omega\left(x^{i}\right)$ and a total localization domain $\tilde{\omega}^{i}$ associated with a collocation point $x^{i}$ of a body $\Omega$ for (a) mesh-based and (b) mesh-less discretizations.

intersect with $\omega\left(x^{i}\right)$ be called the total localization domain $\tilde{\omega}^{i}$, Fig. 2(a). Evidently the closure $\bar{\omega}\left(x^{i}\right) \cap \bar{\Omega}$ belongs to $\tilde{\omega}^{i}$. If $\omega\left(x^{i}\right)$ is sufficiently small, then $\tilde{\omega}^{i}$ consists only of the elements adjacent to the collocation point $x^{i}$. If $\omega\left(x^{i}\right)$ is ab initio chosen as consisting only of the elements adjacent to the collocation point $x^{\mathrm{i}}$, then $\tilde{\omega}^{i}=\bar{\omega}\left(x^{i}\right)$. Let $\dot{u}\left\{\tilde{\omega}^{i}\right\}$ be the array of the function values $\dot{u}\left(x^{j}\right)$ at the node points $x^{j} \in \tilde{\omega}^{i}$ and $J_{\tilde{\omega}^{i}}$ be the number of those node points.

Let $\dot{u}(x)=\sum_{j} \dot{u}\left(x^{j}\right) \phi_{k j}(x)$ be a continuous piece-wise smooth interpolation of $\dot{u}(x)$ at any point $x \in \Omega$ along the values $\dot{u}\left(x^{j}\right)$ at the node points $x^{j}$ belonging to the same element $\bar{e}_{k} \subset \Omega$ as $x$, and the shape functions $\phi_{k j}(x)$ be localized on $\bar{e}_{k}$. Collecting the interpolation formulae, we have for any $x \in \tilde{\omega}^{i}$,

$$
\begin{aligned}
& \dot{u}(x)=\sum_{x^{j} \in \tilde{\omega}^{i}} \dot{u}\left(x^{j}\right) \Phi_{j}(x), \\
& \Phi_{j}(x)= \begin{cases}\phi_{k j}(x) & \text { if } x, x^{j} \in \bar{e}_{k} \\
0 & \text { otherwise }\end{cases}
\end{aligned}
$$

$$
\begin{aligned}
& \nabla \dot{u}(x)=\sum_{x^{j} \in \tilde{\omega}^{i}} \dot{u}\left(x^{j}\right) \nabla \Phi_{j}(x), \\
& \nabla \Phi_{j}(x)= \begin{cases}\nabla \phi_{k j}(x) & \text { if } x, x^{j} \in \bar{e}_{k} \\
0 & \text { otherwise }\end{cases}
\end{aligned}
$$

Consequently, $\Phi_{j}(x)=\nabla \Phi_{j}(x)=0$ if $x \in \tilde{\omega}^{i}$ but $x^{j} \notin \tilde{\omega}^{i}$.

Since interpolation (29) is piece-wise smooth, expressions (30) deliver non-unique values for $\nabla \dot{u}(x)$ on the element interfaces and particularly at the apexes $x^{i}$ of different adjoint elements $e_{k}$. This brings no complications for the choice of the reference elastic moduli as $a_{i j k l}^{*}(y)=a_{i j k l}^{e}(y)$ since they and consequently all other asterisk variables and operators do not depend on $\nabla \dot{u}(y)$, which then appears either in the domain integrals or in the boundary integrals with the gradients taken from the corresponding side of the boundary. On the other hand, for the reference elastic tensor chosen as the current tangent tensor, $a_{i j k l}^{*}(y)=a_{i j k l}(\nabla \dot{u}(y), y)$, one has to estimate $\nabla u(y)$ to calculate the coefficient $a(\nabla \dot{u}(y), y)$ and, consequently $\tilde{a}(\nabla \dot{u} ; x, y), T^{(y)^{*}}(u), P^{(y)^{*}}(u ; x, y)$ and $R^{(y)^{*}}(u ; x, y)$ at the collocation points $y=x^{i}$. A possible way out is to assign 


$$
\begin{aligned}
& \nabla u\left(x^{i}\right):=\sum_{\bar{e}_{k} \ni x^{i}} \frac{\alpha_{k}\left(x^{i}\right)}{\alpha\left(x^{i}\right)} \nabla u^{k}\left(x^{i}\right), \\
& \nabla u^{k}\left(x^{i}\right):=\sum_{x^{j} \in \bar{e}_{k}} u\left(x^{j}\right) \nabla \phi_{k j}\left(x^{i}\right),
\end{aligned}
$$

where $\alpha\left(x^{i}\right)$ is an interior space angle at the apex $x^{i}$ of the element $e_{k}$ and $\alpha\left(x^{i}\right)=\sum_{\bar{e}_{k} \ni x^{i}} \alpha_{k}\left(x^{i}\right)$.

For discretization of the partly segregated LBDIDE, we can also use a local interpolation of the unknown flux variable $\tilde{t}(x)$ along only boundary nodes belonging to $\tilde{\omega}^{i} \cap \partial_{D} \Omega$,

$\tilde{t}(x)=\sum_{x^{j} \in \tilde{\omega}^{i} \cap \partial_{\mathrm{D}} \Omega} \tilde{t}\left(x^{j}\right) \Phi_{j}^{\prime}(x), \quad x \in \tilde{\omega}^{i} \cap \partial_{\mathrm{D}} \Omega$.

Here $\Phi_{j}^{\prime}(x)$ are the shape functions on the boundary obtained similar to $\Phi_{j}(x)$ in (29).

\subsubsection{Mesh-based discretization of the partly segregated $\angle B D I D E$}

After substituting the above interpolations in LBDIDE (27) of the partly segregated formulation at the collocation points $y=x^{i} \in \bar{\Omega}$, and taking into account (11), we derive the following system of $J \times n$ quasi-linear algebraic equations for $J \times n$ unknowns: $\dot{u}\left(x^{j}\right), x^{j} \in \Omega \cup \partial_{\mathrm{N}} \Omega$ and $\tilde{t}\left(x^{j}\right), x^{j} \in \partial_{\mathrm{D}} \Omega$,

$$
\begin{aligned}
& c^{0}\left(x^{i}\right) \dot{u}\left(x^{i}\right)+\sum_{x^{j} \in \tilde{\omega}^{i} \backslash \partial_{\mathrm{D}} \Omega} K_{i j}^{0}\left(\dot{u}\left\{\tilde{\omega}^{i}\right\}\right) \dot{u}\left(x^{j}\right) \\
& +\sum_{x^{j} \in \partial_{\mathrm{D}} \Omega \cap \tilde{\omega}^{i}} Q_{i j}\left(\dot{u}\left\{\tilde{\omega}^{i}\right\}\right) \tilde{t}\left(x^{j}\right) \\
& =\mathcal{F}_{\omega}^{0}\left(x^{i}\right)-\sum_{x^{j} \in \partial_{\mathrm{D}} \Omega \cap \tilde{\omega}^{i}} K_{i j}^{0}\left(\dot{u}^{i}\left\{\tilde{\omega}^{i}\right\}\right) \check{u}\left(x^{j}\right), \quad x^{i} \in \bar{\Omega}
\end{aligned}
$$

(no sum in $i$ ). For fixed indices $i, j$, the $n \times n$ tensors $K_{i j}^{0}\left(\dot{u}_{\{}\left\{\tilde{\omega}^{i}\right\}\right.$ ) and $Q_{i j}\left(\dot{u}\left\{\tilde{\omega}^{i}\right\}\right)$ are

$$
\begin{aligned}
K_{i j}^{0}\left(\dot{u}\left\{\tilde{\omega}^{i}\right\}\right)= & -\int_{\bar{\omega}\left(x^{i}\right) \cap \partial_{N} \Omega} \Phi_{j}(x)\left[T^{\left(x^{i}\right) *} P_{\omega}^{\left(x^{i}\right) *}\left(\cdot, x^{i}\right)\right](x) \mathrm{d} \Gamma(x) \\
& -\int_{\Omega \cap \partial \omega\left(x^{i}\right)} \Phi_{j}(x)\left[T^{\left(x^{i}\right) *} P_{\omega}^{\left(x^{i}\right) *}\left(\cdot, x^{i}\right)\right](x) \mathrm{d} \Gamma(x) \\
& +\int_{\Omega \cap \partial \omega\left(x^{i}\right)} P_{\omega}^{\left(x^{i}\right) *}\left(x, x^{i}\right)\left[T\left(\dot{u}\left\{\tilde{\omega}^{i}\right\}\right) \Phi_{j}\right](x) \mathrm{d} \Gamma(x) \\
& -\int_{\omega\left(x^{i}\right) \cap \Omega}\left[\nabla^{(x)} P_{\omega}^{\left(x^{i}\right) *}\left(x, x^{i}\right)\right] \tilde{a}\left(\dot{u}^{\prime}\left\{\tilde{\omega}^{i}\right\} ; x, x^{i}\right) \nabla \Phi_{j}(x) \mathrm{d} \Omega(x) \\
& +\int_{\omega\left(x^{i}\right) \cap \Omega} R_{\omega}^{\left(x^{i}\right) *}\left(x, x^{i}\right) \Phi_{j}(x) \mathrm{d} \Omega(x) .
\end{aligned}
$$

$$
Q_{i j}\left(\dot{u}\left\{\tilde{\omega}^{i}\right\}\right)=\int_{\bar{\omega}\left(x^{i}\right) \cap \partial_{D} \Omega} P_{\omega}^{\left(x^{i}\right) *}\left(x, x^{i}\right)\left[T\left(\dot{u}\left\{\tilde{\omega}^{i}\right\}\right) \Phi_{j}\right](x) \mathrm{d} \Omega(x),
$$

(no sum in $i$ ).

Note that the term with $R_{\omega}^{\left(x^{i}\right) *}$ disappears in the last integral in the right hand side of (34) if the parametrix $P_{\omega}^{\left(x^{i}\right) *}\left(x, x^{i}\right)$ is given by (22). On the other hand, if the cut-off function $\chi\left(x, x^{i}\right)$ and its normal derivative are equal zero at $x$ on the boundary $\partial \omega\left(x^{i}\right)$, then the second integral (along $\Omega \cap \partial \omega\left(x^{i}\right)$ ) disappears in the right hand side of (34).

\subsubsection{Mesh-based discretization of the united LBDIDE}

Substituting interpolation formulae (29) and (30) in LBDIDE (25), we arrive at the following system of $J \times n$ quasi-linear algebraic equations for $J \times n$ unknowns $\dot{u}_{m}\left(x^{j}\right)$, $x^{j} \in \bar{\Omega}, m=1, \ldots, n$,

$c\left(x^{i}\right) \dot{u}\left(x^{i}\right)+\sum_{x^{j} \in \tilde{\omega}^{i}} K_{i j}\left(\dot{u}\left\{\tilde{\omega}^{i}\right\}\right) \dot{u}\left(x^{j}\right)=\mathscr{F}_{\omega}\left(x^{i}\right)$,

$x^{i} \in \bar{\Omega}, \quad$ no sum in $i$.

Here

$$
\begin{aligned}
K_{i j}\left(\dot{u}\left\{\tilde{\omega}^{i}\right\}\right)= & K_{i j}^{0}\left(\dot{u}\left\{\tilde{\omega}^{i}\right\}\right)+\int_{\bar{\omega}\left(x^{i}\right) \cap \partial_{D} \Omega} P_{\omega}^{\left(x^{i}\right) *}\left(x, x^{i}\right) \\
& \times\left[T\left(\dot{u}\left\{\tilde{\omega}^{i}\right\}\right) \Phi_{j}\right](x) \mathrm{d} \Gamma(x),
\end{aligned}
$$

(no sum in $i$ ).

The approximate tangent stiffness tensor $a\left(\dot{u}\left\{\tilde{\omega}^{i}\right\} ; x\right)$ and consequently $\tilde{a}\left(\dot{u}\left\{\tilde{\omega}^{i}\right\} ; x, x^{i}\right)$ and the traction operator $T\left(\dot{u}\left\{\tilde{\omega}^{i}\right\}\right)$ in (34), (35) and (37) are expressed in terms of the set of unknowns $\dot{u}\left\{\tilde{\omega}^{i}\right\}:=\left\{\dot{u}\left(x^{j}\right), x^{j} \in \tilde{\omega}^{i}\right\}$. The expressions are obtained after substituting interpolation formulae (30) for $\nabla \dot{u}$ $(x)$ in the coefficient $a(\nabla \dot{u}(x), x)$ in the definitions for $\tilde{a}(\nabla \dot{u} ; x, y)$ and $T(\dot{u})$. In fact, the coefficients also depend on the strainstress history (assumed to be already known) at each point. For the choice $a^{*}(y)=a(\nabla \dot{u}(y), y)$, the asterisk functions and operators depend on $a^{*}\left(\nabla \dot{u}\left(x^{i}\right), x^{i}\right)$ and thus on $\nabla \dot{u}\left(x^{i}\right)$, which is expressed by interpolation formula (31) in terms of $\dot{u}\left\{\tilde{\omega}^{i}\right\}$.

\subsection{Mesh-less discretization}

\subsubsection{Mesh-less approximation}

For a mesh-less discretization, one needs a method of local interpolation or approximation of a function along randomly distributed nodes $x^{i}$. We will suppose all the approximation nodes $x^{i}$ belong to $\bar{\Omega}$ and will use them also as collocation points for the LBDIDE discretization. Let, as before, $J$ be the total number of nodes $x^{i}(i=1,2, \ldots, J)$ in $\bar{\Omega}$, from which $J_{\mathrm{D}}$ nodes are posed on the boundary $\partial_{\mathrm{D}} \Omega$. Let us consider a meshless method, for example, the moving least squares (MLS) (see e.g. $[16-19,25])$, that leads to the following approximation of a function $\dot{u}(x)$

$\dot{u}(x)=\sum_{x^{j} \in \omega_{0}(x)} \hat{u}\left(x^{j}\right) \Phi_{j}(x), \quad x \in \Omega$. 
Here $\Phi_{j}(x)$ are known smooth shape functions such that $\Phi_{j}(x)=$ 0 if $x^{j} \notin \omega_{0}(x), \omega_{0}(x)$ is a localization domain of the approximation formula, and $\hat{u}\left(x^{j}\right)$ are unknown values of an auxiliary function $\hat{u}(x)$ at the nodes $x^{j}$, that is, the so-called $\delta$-property is not assumed for approximation (38).

Let $\omega\left(x^{i}\right)$ be a localization domain around a node $x^{i}$. Then for any $x \in \bar{\omega}\left(x^{i}\right)$, the total approximation of $\dot{u}(x)$ can be written in the following local form,

$\dot{u}(x)=\sum_{x^{j} \in \tilde{\omega}^{i}} \hat{u}\left(x^{j}\right) \Phi_{j}(x), \quad \nabla \dot{u}(x)=\sum_{x^{j} \in \tilde{\omega}^{i}} \hat{u}\left(x^{j}\right) \nabla \Phi_{j}(x)$,

$x \in \bar{\omega}\left(x^{i}\right)$

where $\tilde{\omega}^{i}:=\cup_{x \in \bar{\omega}\left(x^{i}\right) \cap \bar{\Omega}} \omega_{0}(x)$ is a total localization domain, Fig.2(b). Consequently, $\Phi_{j}(x)=\nabla \Phi_{j}(x)=0$ if $x \in \bar{\omega}\left(x^{i}\right)$ and $x^{j} \notin \tilde{\omega}^{i}$. Let $J_{\tilde{\omega}^{i}}$ be the number of nodes $x^{j} \in \tilde{\omega}^{i}$ and $\hat{u}\left\{\tilde{\omega}^{i}\right\}$ be the array of the function values $\hat{u}\left(x^{j}\right)$ at the node points $x^{j} \in \tilde{\omega}^{i}$. Since our approximation (39) for $\dot{u}$ is smooth, its gradient approximation in (39) is continuous, and we do not need special formulae like (31) for calculating gradients $\nabla \dot{u}\left(x^{(i)}\right)$ at the collocation points $x^{(i)}$ if the reference stiffness tensor is chosen as $a^{*}(y)=a(\nabla \dot{u}(y), y)$.

\subsubsection{Mesh-less discretization of the partly segregated $L B D I D E$}

After substitution of approximation (39) in LBDIDE (27) and in the Dirichlet boundary conditions (11), we arrive at the following quasi-linear system of $\left(J+J_{\mathrm{D}}\right) \times n$ algebraic equations with respect to $J \times \mathrm{n}$ unknowns $\hat{u}_{m}\left(x^{j}\right), x^{j} \in \bar{\Omega}, m=$ $1, \ldots, n$, and $J_{\mathrm{D}} \times n$ unknowns $\tilde{t}_{m}\left(x^{j}\right), x^{j} \in \bar{\Omega}, m=1, \ldots, n$,

$$
\begin{aligned}
& \sum_{x^{j} \in \tilde{\omega}^{i}}\left[c^{0}\left(x^{i}\right) \Phi_{j}\left(x^{i}\right)+K_{i j}^{0}\left(\hat{u}\left\{\tilde{\omega}^{i}\right\}\right)\right] \hat{u}\left(x^{j}\right) \\
& +\sum_{x^{j} \in \partial_{\mathrm{D}} \Omega \cap \tilde{\omega}^{i}} Q_{i j}\left(\dot{u}\left\{\tilde{\omega}^{i}\right\}\right) \tilde{t}\left(x^{j}\right)=\mathcal{F}_{\omega}^{0}\left(x^{i}\right), \quad x^{i} \in \bar{\Omega}, \quad \text { no sum in } i .
\end{aligned}
$$

$$
\sum_{x^{j} \in \tilde{\omega}^{i}} \hat{u}\left(x^{j}\right) \Phi_{j}\left(x^{i}\right)=\check{u}\left(x^{i}\right), \quad x^{i} \in \partial_{\mathrm{D}} \Omega .
$$

\subsubsection{Mesh-less discretization of the united LBDIDE}

Alternatively, one can substitute approximation (39) in LBDIDE (25) and arrive at the following system of quasi-linear system of $J \times n$ algebraic equations with respect to $J \times n$ unknowns $\hat{u}_{m}\left(x^{j}\right), x^{j} \in \bar{\Omega}, m=1, \ldots, n$,

$\sum_{x^{j} \in \tilde{\omega}^{i}}\left[c\left(x^{i}\right) \Phi_{j}\left(x^{i}\right)+K_{i j}\left(\hat{u}\left\{\tilde{\omega}^{i}\right\}\right)\right] \hat{u}\left(x^{j}\right)=\mathscr{F}_{\omega}\left(\hat{u}\left\{\tilde{\omega}^{i}\right\}, x^{i}\right)$,

$x^{i} \in \bar{\Omega}$, no sum in $i$.

For any $i, j$, the $n \times n$ tensors $K_{i j}^{0}, Q_{i j}$ and $K_{i j}$ in (40) and (42) are given by expressions (34), (35) and (37), respectively, with the shape functions $\Phi_{j}$ from (39), and $\dot{u}\left\{\tilde{\omega}^{i}\right\}$ replaced by $\hat{u}\left\{\tilde{\omega}^{i}\right\}$. Again, they also depend on the strain-stress history (assumed to be already known) at each point. Expressions for $a\left(\dot{u}\left\{\tilde{\omega}^{i}\right\} ; x\right)$ and consequently for $\tilde{a}\left(\hat{u}\left\{\tilde{\omega}^{i}\right\} ; x, x^{i}\right)$ and $T\left(\hat{u}\left\{\tilde{\omega}^{i}\right\}\right)$ in terms of the set of unknowns $\hat{u}\left\{\tilde{\omega}^{i}\right\}:=\left\{\hat{u}\left(x^{j}\right), x^{j} \in \tilde{\omega}^{i}\right\}$ in (37) are obtained after substituting approximation formulae (39) for $\nabla \dot{u}$ in the coefficient $a(\nabla \dot{u}(x), x)$ in the definitions for $\tilde{a}(\nabla \dot{u} ; x, y)$ and $T(\dot{u})$. For the choice $a^{*}(y)=a(\nabla \dot{u}(y), y)$, the asterisk functions and operators depend on $a^{*}\left(\nabla \dot{u}\left(x^{i}\right), x^{i}\right)$ and thus on $\nabla \dot{u}\left(x^{i}\right)$, which is expressed for the mesh-less approach by the same smooth approximation formula (39) in terms of $\hat{u}\left\{\tilde{\omega}^{i}\right\}$.

\section{Conclusion}

Nonlinear BDIDEs (18) and (19) as well as LBDIDEs (25) and (27) are integro-differential reformulations of elastoplastic BVP (10)-(12). Depending on the choice of the reference elastic tensor $a^{*}$ as the initial elastic or currant tangent stiffness tensor, one can obtain two different versions of the BDIDEs and LBDIDEs. Different strategies can be chosen for the numerical solution of the BDIDEs or LBDIDEs to obtain the complete evolutionary solution of the problem. One of them is to split the process into the time steps $t_{i}$ and solve either of the integral equations with respect to the displacement rate $\dot{u}_{k}\left(x, t_{i}\right)$ employing the necessary stress and strain fields $\sigma\left(x, t_{i}\right), \varepsilon\left(x, t_{i}\right)$ obtained at the previous step. Then one finds the stress rate from (6) and approximate the displacement increment during the time step as $\Delta u_{k}\left(x, t_{i}\right)=$ $\dot{u}_{k}\left(x, t_{i}\right)\left(t_{i+1}-t_{i}\right)$ and strain and stress increments similarly. This allows to calculate the stress and strain fields at time $t_{i+1}$.

While discretizing LBDIDEs (25) and (27), we have from the definitions in both mesh-based and mesh-less methods that $\Phi_{j}(x)=\nabla \Phi_{j}(x)=\left[T(\dot{u}) \Phi_{j}\right](x)=\left[T^{(y) *} \Phi_{j}\right](x)=0$ if $x \in \bar{\omega}\left(x^{i}\right)$ but $x^{j} \notin \tilde{\omega}^{i}$. Consequently $K_{i j}=0, K_{i j}^{0}=0$ and $Q_{i j}=0$ if $x^{j} \notin \tilde{\omega}^{i}$, and moreover, $K_{i j}, K_{i j}^{0}$ and $Q_{i j}$ depend only on $\dot{u}\left\{\tilde{\omega}^{i}\right\}$ or $\hat{u}\left\{\tilde{\omega}^{i}\right\}$, respectively. Thus, each algebraic equation in (33), (36), (40) or (42) has not more than $J_{\tilde{\omega}^{i}} \times n \ll J \times n$ non-zero entries, i.e. the systems are sparse. The number of the nodal points, $J_{\tilde{\omega}^{i}}$ in the total localization domain $\tilde{\omega}^{i}$ depends on the type of the domain elements, the choice of the localization domains and the local interpolation/approximation formulae. If during re-meshing the element types are roughly the same, and the localization domains and the support of the interpolation/ approximation formulas are related with the domain elements near collocation points, then the number of the nonzero entries of each equation, $J_{\tilde{\omega}^{i}} \times n$, will be practically independent of remeshing.

The second kind structure of the nonlinear united LBDIDEs and of the corresponding mesh-based discrete systems look very promising for constructing simple and fast converging iteration algorithms for their numerical solution without preconditioning, thus outperforming other available numerical techniques.

\section{References}

[1] Brebbia CA, Telles JCF, Wrobel LC. Boundary element techniques. Berlin: Springer; 1984.

[2] Balaš J, Sladek J, Sladek V. Stress analysis by boundary element methods. Amsterdam: Elsevier; 1989.

[3] Banerjee PK. The boundary element methods in engineering. London: McGraw-Hill; 1994. 
[4] Aliabadi MH. The boundary element method. Applications in solids and structures, vol. 2. Chichester: Wiley; 2002.

[5] Gao X-W, Davies TG. Boundary element programming in mechanics. Cambridge: Cambridge University Press; 2002.

[6] Mikhailov SE. Localized boundary-domain integral formulations for problems with variable coefficients. Eng Anal Bound Elem 2002;26: 681-90.

[7] Mikhailov SE, Nakhova IS. Numerical solution of a Neumann problem with variable coefficients by the localized boundary-domain integral equation method. In: Amini S, editor. Fourth UK conference on boundary integral methods. The Crescent: Salford University; 2003. p. 175-84.

[8] Mikhailov SE, Nakhova IS. Mesh-based numerical implementation of the localized boundary-domain integral equation method to a variablecoefficient Neumann problem. J Eng Math 2005;51:251-9.

[9] Sladek V, Sladek J. A new formulation for solution of boundary value problems using domain-type approximations and local integral equations. Electron J Bound Elem 2003;1:132-53.

[10] Sladek J, Sladek V, Zhang Ch. Local integro-differential equations with domain elements for the numerical solution of partial differential equations with variable coefficients. J Eng Math 2005;51:261-82.

[11] Mikhailov SE. About localized boundary-domain integro-differential formulations for a quasi-linear problem with variable coefficients. In: Constanda C, Ahues M, Largillier A, editors. Integral methods in science and engineering: analytic and numerical techniques. Berlin: Birkhäuser; 2004. p. 139-44.

[12] Mikhailov SE. Localized direct boundary-domain integro-differential formulations for scalar nonlinear problems with variable coefficients. J Eng Math 2005;51:283-302.

[13] Mikhailov SE. Some localized boundary-domain integro-differential formulations for quasi-linear problems with variable coefficients. In: Gallego R, Aliabadi MH, editors. Advances in boundary element techniques IV. Queen Mary: University of London; 2003. p. 289-94.

[14] Mikhailov SE. Localized boundary-domain integro-differential formulation for physically nonlinear elasticity of inhomogeneous body. In: Leitao VMA, Aliabadi MH, editors. Advances in boundary element techniques V. UK: EC Ltd; 2004. p. 31-8, ISBN 09547783-0-8.
[15] Mikhailov SE. Direct localized boundary-domain integro-differential formulations for physically nonlinear elasticity of inhomogeneous body. Eng Anal Bound Elem 2005;29:1008-15.

[16] Zhu T, Zhang J-D, Atluri SN. A local boundary integral equation (LBIE) method in computational mechanics, and a meshless discretization approach. Comput Mech 1998;21:223-35.

[17] Zhu T, Zhang J-D, Atluri SN. A meshless numerical method based on the local boundary integral equation (LBIE) to solve linear and nonlinear boundary value problems. Eng Anal Bound Elem 1999;23: 375-89.

[18] Sladek J, Sladek V, Atluri SN. Local boundary integral equation (LBIE) method for solving problems of elasticity with nonhomogeneous material properties. Comput Mech 2000;24:456-62.

[19] Sladek J, Sladek V, Zhang Ch. A local BIEM for analysis of transient heat conduction with nonlinear source terms in FGMs. Eng Anal Bound Elem 2004;28:1-11.

[20] Mikhailov SE. Localized boundary-domain integro-differential formulation for incremental elasto-plasticity of inhomogeneous body. In: Yao ZH, Yuan MW, Zhong WX, editors. Computational mechanics, WCCM VI in conjunction with APCOM'04, Sept. 5-10, 2004. Beijing, China: Tsinghua University Press/Springer; 20047-89494-5129. $9 \mathrm{p}$.

[21] Synge JL. Hypercircle in mathematical physics. Cambridge: Cambridge University Press; 1957.

[22] Vogel SM, Rizzo FJ. An integral equation formulation of threedimensional anisotropic boundary value problems. J Elasticity 1973;3: 203-16.

[23] Wilson RB, Cruse TA. Efficient implementation of anisotropic threedimensional boundary integral equation stress analysis. Int J Numer Methods Eng 1978;12:1383-97.

[24] Miranda C. Partial differential equations of elliptic type. Berlin: Springer; 1970.

[25] Belytschko T, Krongauz Y, Organ D, Flemming M, Krysl P. Meshless methods: An overview and recent developments. Comput Methods Appl Mech Eng 1996;139:4-47. 